\title{
Langerhans cell histiocytosis following treatment for testicular cancer. A case report and literature review
}

\author{
Pistamaltzian Nikolaos*, Nikolaidi Adamantia, Raftogiannis Maria, \\ Economou Aggelos, Mourtzoukos Spryridon, Athanasiadis llias \\ 2nd Oncology Department, MITERA Hospital, 6 Erythrou Stavrou Street, \\ 15123 Marousi, Greece
}

\begin{abstract}
A 35 year old male patient received treatment for testicular cancer of pure seminoma histology. He underwent initially a right inguinal orchiectomy and afterwards he received 3 cycles of BEP chemotherapy, as his imaging studies showed enlarged para-aortic lymph nodes. Five months after completion of chemotherapy treatment, a thoracic CT revealed multiple micronodular lesions in both lungs. The patient was advised about the need of salvage chemotherapy, but he opted to undergo further investigation. A lung lesion biopsy was performed, and histology was compatible with diagnosis of Langerhans cell histiocytosis (LCH).

LCH is quite an unusual clinical entity that affects usually -but not solely- the lungs of young adults. The hallmark of the disease, is the presence of the activated Langerhans cells. As its clinical, laboratory and radiological findings are nonspecific, a transbronchial biopsy is sometimes necessary to establish diagnosis. Treatment is generally symptomatic and reserved for patients with multisystem organ involvement.

LCH's relationship with cancer is complex and no certain correlation is evident. More troublesome can be its differential diagnosis from a cancer with metastatic spread to the lungs or other affected organs, and although rare, it has to be kept in mind when suspicious signs arise

Keywords: Langerhans cell histiocytosis • Testicular cancer • Chemotherapy • Lung nodules

(c) De Gruyter Open
\end{abstract}

\section{Case report}

A 35-year-old male patient was urgently admitted to our clinic in March 2013, due to colicky pain at the right lumbar site, radiating to the right inguinal area. An abdominal ultrasound revealed enlarged para-aortic lymph nodes, a significant dilatation of the right kidney's pelvicalyceal system and a cystic formation arising from the scrotum. At the periphery of the scrotal cyst, a solid multilobular lesion was apparent, along with a sufficient quantity of liquid, with a maximum diameter of $12 \mathrm{~cm}$ within the scrotum. Levels for LDH, $\beta$-hCG and AFP were $558 \mathrm{IU} / \mathrm{l}, 22 \mathrm{mlU} / \mathrm{ml}, 13 \mathrm{ng} / \mathrm{ml}$, respectively. Chest X-ray was unremarkable for pathological findings, whereas the full dynamic spirometry was normal. An abdominal and pelvic MRI performed thereafter confirmed that the lesion is in contact with the right testis and the rest of the aforementioned ultrasound results (images 1-2).
The patient underwent a right inguinal orchiectomy on 30/03/2013. The histologic examination of the specimen revealed a pure seminoma of the testis. The immunohistochemistry was positive for PLAP and CD117 and negative for AFP, b-HCG, AE1/AE3 and CD30 stains. Forty percent of the neoplastic cells showed nuclear positivity over the proliferation index Ki67. A normal heart triplex with an ejection fraction of $65 \%$ was confirmed before the beginning of chemotherapy. The patient was then treated with 3 BEP chemotherapy cycles (cisplatin $20 \mathrm{mg} / \mathrm{m}^{2} \mathrm{~d} 1-\mathrm{d} 5$, etoposide $100 \mathrm{mg} / \mathrm{m}^{2}$ d1-d5 and bleomycin 30 MIU on d2, d9, d16 in 21 days cycles), from 02/04/2013 to 28/05/2013.

After the completion of chemotherapy, the patient underwent a reevaluation on 08/05/2013 with thoracic and abdominal CTs to evaluate the response to the therapy. According to the results, no pathological findings were found in the thorax, whereas in the abdomen, there 


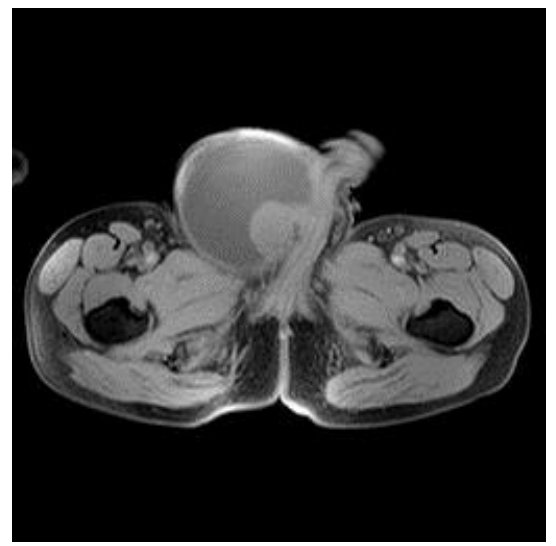

Image 1.

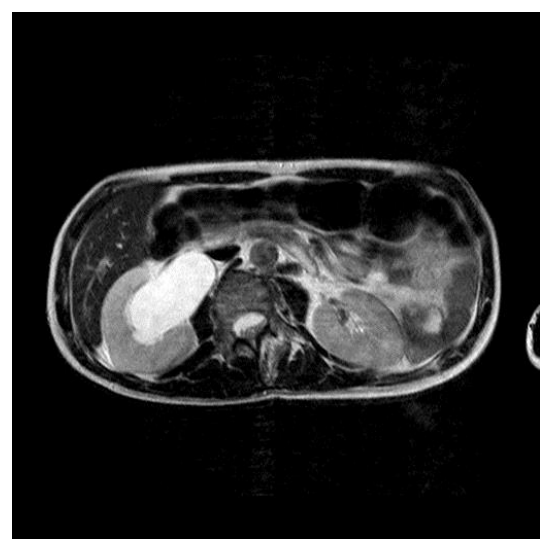

\section{Image 2.}

were only postoperative lesions in the right scrotal area, with a significant remission of the lesions in the right spermatic cord and around the distal portion of the ipsilateral renal vein, an almost total regression of the dilatation of the right kidney's pelvicalyceal system and a reduction of the size of the two para-aortic lymph nodes (image 3).

The patient performed his next programmed evaluations in July 2013 and October 2013. The clinical examination was normal. The thoracic and abdominal CTs of July were unremarkable for any findings. Upon October's reevaluation, there was a mild dilatation of the right pelvicalyceal system, without an apparent cause of obstruction, whereas the thoracic CT revealed many micronodular lesions in both lungs (images 4-5), with an increase in the values of $\mathrm{LDH}, \mathrm{b}-\mathrm{HCG}$ and AFP. Because of the likelihood of relapsed disease, the patient was advised about the need for salvage chemotherapy, but he opted to undergo further investigation first, and thus one of the lung lesions was excised. According to the histologic examination, the lesion was compatible with Langerhans cell histiocytosis.

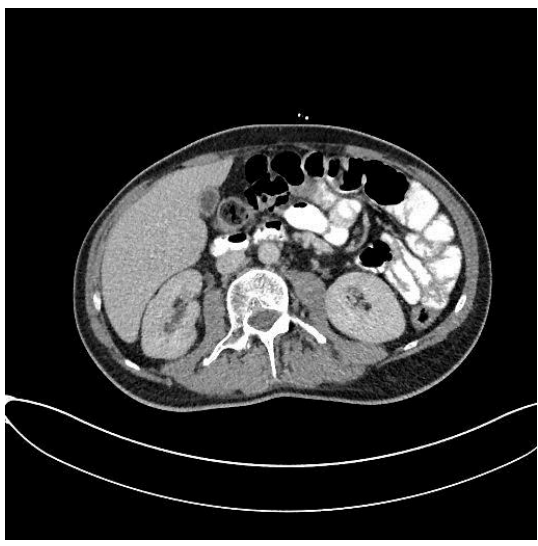

Image 3.

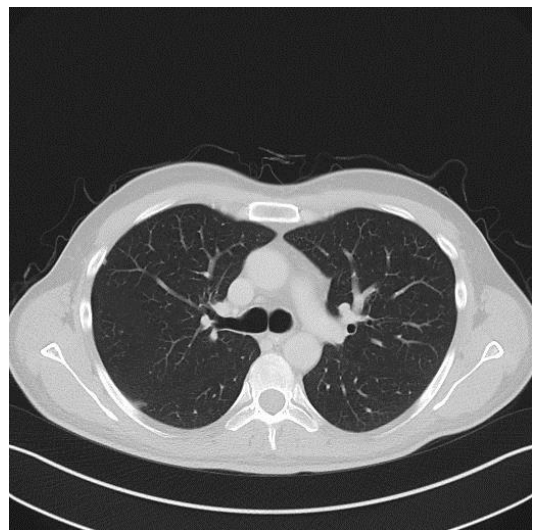

Image 4.

\section{Discussion}

Pulmonary Langerhans histiocytosis, also known as eosinophlic granuloma or pulmonary histiocytosis $(\mathrm{PLCH}) \mathrm{X}$, is a rare idiopathic disorder that mainly appears in young smokers, in ages ranging from 20 to 40 years. The lungs are usually the only affected organ and upon histologic examination, infiltration and destruction of peripheral bronchioles by Lagerhans granulomas is evident (1).

The diagnosis is based upon high-resolution CT, where solid or cystic nodules and cysts with thin or thick walls are apparent. The identification of the disease is confirmed by the presence of Langerhans-type cells in a lung biopsy. No effective treatment of the disease seems to exist (2).

Langerhans cell histiocytosis ( $\mathrm{LCH}$ ) includes a group of disorders classified as: i) acute diffuse LCH (disease Letterer-Siwe), a serious polysystemic disease, ii) multifocal LCH (syndrome Hand-Schueller-Christian or multifocal eosinophilic granuloma) and iii) PLCH (3). 


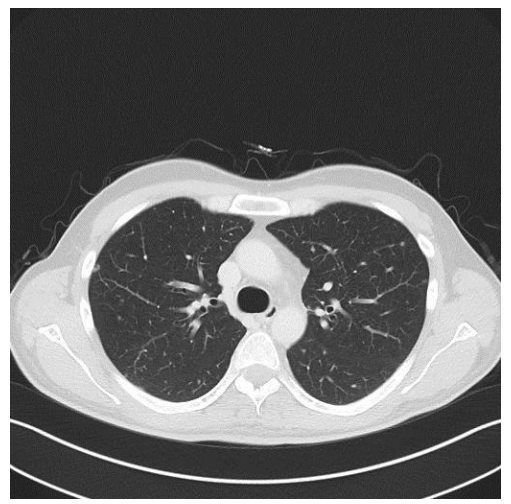

Image 5.

As far as PLCH is concerned, its appearance is occasional. The widespread use of HRCT has aided in the quicker diagnosis and therefore, an increase in the number of the mentioned cases. The symptoms may be mild or even totally absent. In approximately one-fourth of the total cases, the disease is detected in a random routine thoracic X-ray. In two-thirds of the cases, the main symptoms arise from the respiratory system, and are mainly dry cough and dyspnea during exercise; sometimes, there are non-specific symptoms such as fever, night sweating, weakness and loss of weight. Finally, in $10-20 \%$ of the cases, spontaneous pneumothorax is the first symptom and eventually, leads to the final diagnosis (4).

PLCH mainly affects only one of the two lungs, whereas in most of the cases, it rarely affects other organs besides the lung. The most common extrapulmonary manifestations are diabetes insipidus, skin lesions and skeletal-related events depending upon the affected organ (5).

Laboratory findings are non-specific. Transbronchial lung biopsy sometimes leads to the correct diagnosis, although in the USA, the reported sensitivity of this particular method is in the range of $10-40 \%$. The detection of Langerhans cells in bronchoalvelolar lavage is performed with the use of monoclonal antibodies against CD1a (6).

Upon histologic examination, the hallmark of the disease is the presence of the activated Langerhans cells. The cells are organised in granulomas and infiltration of the distal bronchioles is apparent. Langerhans cells are dendritic cells originating form the bone marrow. Inside the lesion, other leukocytic subpopulations such as lymphocytes, eosinophils, macrophages and neutrophils can also be found. The lesions appear focal, surrounded by healthy parenchyma and are located in the distal bronchioles, resulting in the destruction of the airways' walls. The granulomas are not entrenched and may extend to adjacent alveoli, creating an image that resembles with that of interstitial lung disease. In non-affected areas, the architecture of pulmonary parenchyma remains intact. Gradually, as Langerhans cells are decreasing, lymphocytes and other inflammatory cells, including eosinophils, macrophages and a small number of polymorphonuclear leukocytes are increasing. In the final phase of the disease, there is fibrosis of the lesions and subsequent formation of cavities and this is the pathologic mechanism behind the characteristic honeycomb motif of the lung that can be seen at that point (7).

Smoking seems to be the major predisposing factor, although cases after chemotherapy or radiotherapy in patients with lymphoma, mainly of Hodgkin's type, have also been reported $(\mathbf{8}, \mathbf{9})$.

$\mathrm{PLCH}$ does not seem to share the same biological characteristics with malignant neoplasms, but rather it seems to be a hyper-responsiveness state where there is a disturbance of the balance between proliferative and apoptotic mechanisms (10).

The relationship with cancer has to do mainly with the simultaneous appearance of PLCH and cancer, and it has been reported that when the neoplasm is treated either with surgery or systemic therapy, PLCH recession also occurs. In some cases, the simultaneous appearance of PLCH and cancer might be completely random or there could be a reactive correlation, as it happens with lymphoma cases or there may be a causal relationship with other factors that are also associated with carcinogenesis (e.g. smoking and lung carcinomas) $(11,12)$. It seems that there is a predisposition to PLCH in individuals with impaired immunity, and this is linked to ineffective immunosurveillance mechanisms and deregulated properties of the immune system. This is possibly attributed to a clonal disorder that is associated in some cases with a BRAF mutation (13).

As it concerns the demographics of the disease, the heterochronous and mainly the metachronous appearance of PLCH, in conjunction with malignancy (in $78 \%$ of cases, the diagnosis of PLCH precedes) appears to be quite rare. Also, the majority $(78 \%)$ of patients with simultaneous appearance of $\mathrm{LCH}$ and solid tumours are under the age of 18 years. The disease occurs mainly in children, and more specifically, after therapy containing agents such as methotrexate, vinblastine, cyclophosphamide and etoposide (14).

From our patient's history, there are some precipitating factors that can be identified: he was a smoker who has been exposed to cytotoxic agents such as bleomycin and etoposide. Since he experienced no symptoms, no specific therapy was administered to him. Shortly after the pathology report, a repeat in the measurement of tumour markers revealed normal 
values. We could not identify any specific cause that can justify this temporary rise in their values. Upon his next imaging evaluation in February 2014, the lesions appeared to have an almost complete remission. From our perspective, no causal correlation to cancer can be concluded, but a temporary deregulation of immunosurveillance cannot be ruled out.

\section{References}

[1] Chon S, Kyung SY, Lee SP, Park JW, Jeong SH, Choi SJ, Ha SY. A case of pulmonary Langerhans' cell histiocytosis mimicking hematogenous pulmonary metastases. Korean J Intern Med 2009; 24: 393-396

[2] Fleming CM, Shepard JAO, Mark EJ.: Case 152003: A 47-year old man with waxing and waning pulmonary nodules five years after treatment for testicular seminoma. N Engl J Med 2003; 348: 2019-2027

[3] Arico M. Langerhans cell histiocytosis in adults: more questions than answers? Europ J Cancer 2004; 40: 1467-1473

[4] Willis B, Ablin A, Weinberg V, Zoger S, Wara WM, Matthay KK.: Disease course and late sequale of Langerhans' cell histiocytosis: 25-year experience at the University of California, San Fransisco. J Clin Oncol 1996; 14: 2073-2082

[5] Fulks L, Kramer MR, Shiltrit D, Raviv Y.: Pulmonary Langerhans cell histiocytosis and diabetes insipidus in pregnant women: our experience. Lung 2014; 192:285-287.

[6] Brownlee NA, Mahar A, Sporn TA. Bilateral pulmonary nodules in a 37-year-old woman with malignant melanoma. Arch Pathol Lab Med 2005; 129: e136-137

[7] Willman CL, Busque L, Griffith BB, Favara BE, McClain KL, Duncan MH, Gilliland DG.: Langerhans' cell histiocytosis (Histiocytosis $\mathrm{X}$ ) - A clonal proliferative disease. N Engl J Med 1994; 331:154-160
$\mathrm{LCH}$ diagnosis can be troublesome in cases of patients with a history of malignancy, because its radiologic characteristics can easily be misinterpreted as those of a relapsed disease. Although its appearance is extremely rare, we have to consider this entity in our differential diagnosis work-up plan.

[8] Dehkordi NR, Rajabi P, Naimi A, Heidarpour M. Langerhans cell histiocytosis following Hodgkin lymphoma: a case report from Iran. J Res Med Sci 2010; 15(1): 58-61

[9] Feuillet S, Louis L, Bergeron A, Berenze A, Dubreuil M-L, Polivka M, Oksenhendler E, Tazi A. Pulmonary Langerhans cell histiocytosis associated with Hodgkin's lymphoma. Eur Respir Rev 2010; 19: 115, 86-88

[10] Ng WK, Lam KY, Ng IOL. Langerhans' cell histiocytosis: possible association with malignant germ cell tumors. J Clin Patol 1995; 48: 963-965

[11] Jamaati HR, Shadmehr MB, Saidi B, Khosvari A, Arab M, Mohammadi F. Langerhans Cell Histiocytosis of the Lung and Thyroid, Co-existing with Papillary Thyroid Cancer. Endocr Pathol 2009; 20: 133-136

[12] Tsuji T, Nakamura S, Tanaka M. Pulmonary Langerhans cell histiocytosis associated with lingual carcinoma. Internal Medicine 2004; 8: 713-717

[13] Yousem SA, Dacic S, Nikiforov YE, Nikiforova M. Pulmonary Langerhans Cell Histiocytosis. Profiling of multifocal tumors using next-generation sequencing identifies concordant occurrence of BRAF V600E mutations. Chest 2013; 143(6): 1679-1684

[14] Egeler RM, Neglia JP, Puccetti DM, Brennan CA, Nesbit ME. Association of Langerhans Cell Histiocytosis with malignant neoplasms. Cancer 1993; 71: 865-873 